\title{
Cost effectiveness of abdominal aortic aneurysm screening and rescreening in men in a modern context: evaluation of a hypothetical cohort using a decision analytical model
}

\author{
(c) $\frac{(1)(8)}{\mathrm{gy}}$ MPEN ACCESS
}

\author{
Rikke Søgaard associate professor ${ }^{1}$, Jesper Laustsen chief vascular surgeon ${ }^{2}$, Jes S Lindholt \\ professor $^{34}$
}

${ }^{1}$ Centre for Applied Health Services Research and Technology Assessment (CAST), Institute for Public Health, University of Southern Denmark, 5000 Odense, Denmark; ${ }^{2}$ Department of Cardiothoracic and Vascular Surgery, Aarhus University Hospital, Aarhus, Denmark; ${ }^{3}$ Vascular Research Unit, Viborg Hospital, Viborg, Denmark; ${ }^{4}$ Department of Cardiothoracic and Vascular Surgery, Odense University Hospital, Odense, Denmark

\begin{abstract}
Objective To assess the cost effectiveness of different screening strategies for abdominal aortic aneurysm in men, from the perspective of a national health service.

Setting Screening units at regional hospitals.

Participants Hypothetical cohort of 65 year old men from the general population.

Main outcome measures Costs ( $£$ in 2010) and effect on health outcomes (quality adjusted life years (QALYs)).

Results Screening seems to be highly cost effective compared with not screening. The model estimated a $92 \%$ probability that some form of screening would be cost effective at a threshold of $£ 20000$ ( $€ 24790$; $\$ 31460$ ). If men with an aortic diameter of $25-29 \mathrm{~mm}$ at the initial screening were rescreened once after five years, 452 men per 100000 initially screened would benefit from early detection, whereas lifetime rescreening every five years would detect 794 men per 100000 . We estimated the associated incremental cost effectiveness ratios for rescreening once and lifetime rescreening to be $£ 10013$ and $£ 29680$ per QALY, respectively. The individual probability of being the most cost effective strategy was higher for each rescreening strategy than for the screening once strategy (in view of the $£ 20000$ threshold).

Conclusions This study confirms the cost effectiveness of screening versus no screening and lends further support to considerations of rescreening men at least once for abdominal aortic aneurysm.
\end{abstract}

\section{Introduction}

Screening for abdominal aortic aneurysms has been the subject of intensive focus over the past two decades. It is a classic example of a scenario that seems to satisfy the World Health Organisation screening criteria, because mortality can be reduced if a simple test is performed. ${ }^{1}$ Long term follow-up data from large randomised controlled trials have established that screening in men older than 65 years can reduce mortality related to abdominal aortic aneurysm by about $50 \% .^{23}$ Also, from the ethical perspective, this form of screening offers a clear balance of good over harm. ${ }^{4}$

Several studies have attempted to extrapolate the benefits and costs of screening to a lifetime perspective using decision analytical models. These studies show a highly efficacious and cost effective technology from the healthcare system perspective. ${ }^{5-10}$ The most recent studies have been critically appraised in a systematic review. ${ }^{11} \mathrm{With}$ one or two exceptions, the review noted that the studies had some limitations with respect to the way the natural history of disease was modelled. Furthermore, the results seemed to be driven by very few original studies; for example, the growth and rupture rates from the Multicentre Aneurysm Screening Study ${ }^{12}$ are used in most models. There are good reasons for the adoption of these rates, since this trial seemed to be a unique, well conducted and high quality study. Nevertheless, if the context or the way we see the biological disease process changes over time, these estimates could ultimately have a date of expiry.

Over the past two decades, the practice of primary and secondary prevention for cardiovascular disease has improved substantially. ${ }^{13}$ The extent to which such contextual changes affect the efficacy of screening is controversial, as noted by a recent clinical review. ${ }^{14}$ This review suggested that smoking 
cessation had a role in the prevalence of abdominal aortic aneurysms; however, it noted that there is currently no definitive evidence on the effect of statins, antihypertensive drugs, and $\beta$ blockers. Although the optimal medical management thus remains unclear, does the efficacy of screening in a context without modern regimens for cardiovascular disease prevention hold true today?

The emergence of additional non-pharmaceutical technologies is also likely to have influenced the efficacy of screening. For example, if the rate of incidental detection of aneurysms increases, it subsequently reduces the number of undetected cases and thereby the rationale for screening. The use of ultrasonography, computed tomography, and magnetic resonance imaging has increased markedly over recent years. ${ }^{15}$

Furthermore, endovascular aorta repair has been rapidly emerging, which has probably influenced the costs of detected aneurysms and possibly the outcomes. ${ }^{16}$

Previous decision models seem to be in complete agreement regarding the optimal form of screening being a one-off scan. However, no model has examined the additional benefit of rescreening individuals with an aortic diameter that approaches the aneurysmal threshold. Recent evidence suggests that these cases have the potential to grow, which would seem to justify rescreening. ${ }^{17}$ Furthermore, it has long been recognised that aneurysm growth and rupture rates increase exponentially with the size of the aneurysm. ${ }^{18}$ This exponential increase contrasts with the existing literature, which presents the disease process as a three step model, in which all aneurysms larger than the surgical threshold size are assumed to have equal growth and rupture rates.

In England, a phased implementation of a national screening programme began in March 2009, and national programme coverage is anticipated by March 2013. ${ }^{19}$ A similar plan has been enacted in Scotland, where implementation will commence in autumn 2012. ${ }^{20}$ The current policy discussion in England and Scotland thus concerns the cost effectiveness of additional rescreening in adjunct to the programmes already being implemented. In many other European countries, a national decision on population screening has not been issued, and the relevant policy debate relates to the cost effectiveness of the optimal form of population screening (with or without rescreening) in a modern day context.

We aimed to assess the expected lifetime costs and benefits of four abdominal aortic aneurysm screening strategies from the perspective of a national health service: no screening, once per lifetime screening, twice per lifetime screening with a five year interval, and lifetime screening every five years. In addition to the evaluation of rescreening strategies, we considered a detailed disease process; used the most recent parameter estimates available or original analyses of the Danish vascular registry and a new Danish screening tria ${ }^{21}$; included the endovascular aortic repair technique; and used microsimulation for detailed epidemiological results of key events, in particular the consequences of rescreening.

\section{Methods}

The model evaluated a hypothetical cohort of 100000 men aged 65 years from the general population. We assigned men to one of the four strategies, and recorded relevant events during their lifetime to estimate the expected costs and quality adjusted life years (QALYs). In addition to this summary, the interventions and methods are described in detail in a technical report. ${ }^{22}$

\section{Model strategies}

We specified two baseline comparisons: no screening, after which follow-up and elective surgery occurred only after incidental detection; and once per lifetime screening, after which individuals were offered screening the year they turned 65 years old. We specified two scenarios of additional rescreening for men with an aortic diameter at $25-29 \mathrm{~mm}$ at the initial screening: rescreening once after five years and rescreening every five years for lifetime, unless an aneurysm was detected and they were referred for follow-up or surgical evaluation.

An abdominal aortic aneurysm was defined as an aortic diameter of at least $30 \mathrm{~mm}$, and indication for elective surgery was defined as an aortic diameter of at least $55 \mathrm{~mm}$ with no contraindications. These definitions were common among all strategies. Detected aneurysms smaller than the threshold for referral to elective surgery were followed at annual $(30-49 \mathrm{~mm})$ or biannual (50-54 $\mathrm{mm}$ ) intervals. If a patient had an aneurysm of at least $55 \mathrm{~mm}$ in aortic diameter and was unfit for surgery, no further action was taken unless the patient became symptomatic or rupture occurred.

\section{Model structure}

The figure $\Downarrow$ details the model structure. Overall, individuals begin in one of eight starting states according to their aortic diameter (along the left side of the figure), and then reside or move to subsequent states during so called Markov cycles. Transitions were determined from probabilities of disease states, and after a number of cycles equivalent to the expected remaining lifespan of a 65 year old man, all individuals end up in the dead state.

We made several simplifying assumptions to maintain transparency. The most important assumptions, although supported from biological evidence, were that aortic diameters of 0-24 mm never become aneurysmal, symptoms and ruptures never occur for aneurysms smaller than $50 \mathrm{~mm}$ in aortic diameter, and that relapse never occurs. Incidental detection of asymptomatic aneurysms occurred in all scenarios (with or without screening) and the model acknowledged this incidental detection. In addition to ruptures, patients with symptomatic aneurysms were immediately referred to acute surgery. This immediate referral reduced the potential for early detection only to those cases that were not incidentally detected and to patients who did not develop symptoms or rupture before they were invited to screening. Lastly, we assumed that elective surgery was provided without significant waiting time.

\section{Model parameters}

The model needed the following categories of parameter estimates related to the disease process: prevalence, growth rates, symptom rates, rupture rates, excess mortality due to non-aneurysm related causes, and mortality rates after rupture and surgical treatment. Apart from rupture rates reported by a systematic review that identified two key references, ${ }^{23}{ }^{24}$ parameter estimates were the result of original analyses composed of a combination of research registries from two Danish screening trials, ${ }^{21}$ the Danish vascular registry, and national registries for causes of death. We determined general population mortality from the most recent national statistics, and quality of life from a representative Danish population survey. ${ }^{25}$

We adapted attendance rates and unit costs from previously reported microcosting studies for Denmark. ${ }^{26}$ The effects of screening were adjusted according to two meta-analyses that concluded that screening marginally reduces non-aneurysm 
related mortality and mortality after elective surgery. ${ }^{22}{ }^{27} \mathrm{We}$ reanalysed non-aneurysm related mortality for this study to avoid double counting aneurysm related mortality. Table $1 \Downarrow$ lists all parameter estimates.

\section{Base case analysis}

Input parameters were entered into the model as probability distributions, to reflect the uncertainty surrounding the mean estimates. A cycle length of six months was specified. We used microsimulation of 100000 individuals to provide detailed epidemiological results for each of the strategies. Count variables (trackers) were defined and used to count the number of key events during lifetime simulations such as detection, symptoms, rupture, and death. We also used the trackers to validate the model against trial observations ${ }^{2}$ and general evidence for the biological process, mortality rates, and age of death (web appendix).

For the cost effectiveness analysis, we used probabilistic sensitivity analysis to propagate the joint uncertainty of individual parameters and alternative scenario sensitivity analysis to explore the effect of alternative model assumptions. We repeated the microsimulation of 100000 people 1000 times. Costs ( $£$ in 2010) and effects were reported in net present values, discounting at an annual rate of $3 \%$ according to Danish practice.

\section{Sensitivity analyses}

Several potential drivers of the cost effectiveness are highly correlated. Therefore, we specified four alternative scenarios for the sensitivity analysis (box).

\section{Results \\ Key events}

Detection of aneurysms will occur regardless of screening. However, health professionals would probably not react to small aneurysms in the same manner as they would to large aneurysms. Assuming that the incidental detection rate was zero in small aneurysms and $12 \%$ per year in aneurysms of $55 \mathrm{~mm}$ or more in diameter, the model predicted that 2469 men per 100 000 screened would be detected with a clinically relevant aneurysm (table $2 \Downarrow$ ). Rescreening once after five years would detect an additional 452 men per 100000 initially screened, whereas rescreening every five years for lifetime would detect 794 men per 100000 initially screened. The number of incidentally detected aneurysms decreased alongside systematic screening, as the number of potentially detectable aneurysms decreased.

The model estimated the number of elective surgeries to increase from 861 to 1390 per 100000 as a result of screening once per lifetime. This number increased to 1496 and 1530 per 100000 , for rescreening once and for rescreening for life, respectively. The corresponding number of acute surgeries reduced from 610 to 382 per 100000 after screening once and fell further to 363 and 360 per 100000 after rescreening once and for life, respectively. Relative risk reductions for acute surgery and rupture associated with repeating the rescreening regimen for lifetime were moderate because aneurysms detected later in life seemed to be in size categories, with insignificant symptom and rupture rates.

The rate of aneurysm related mortality reduced from 788 to 538 per 100000 men as a result of screening and fell further to 520 and 511 per 100000 , if screening was repeated after five years or at five year intervals for lifetime, respectively. This decrease was the result of replacing acute surgery with elective surgery, which is characterised by much lower mortality rates after surgery and the prevention of ruptures leading to death before patients reach the hospital. Non-aneurysm related mortality represents the residual lifetime mortality and, thus, varies only if the aneurysm related mortality occurred first. Estimated age at aneurysm related death increased after screening was provided, but then fell when rescreening was provided-although fewer men would die, relatively more deaths were caused by elective surgery, which decreases in the early cycles of the model.

Table $3 \Downarrow$ details the findings after rescreening. Rescreening men with an aortic diameter of $25-29 \mathrm{~mm}$ at the initial screening led to the detection of an additional 452 and 794 men with clinically relevant aneurysms per 100000 initially screened, for rescreening once after five years and at five year intervals for life, respectively. Furthermore, most of those clinically relevant aneurysms additionally detected were smaller than the threshold for surgery referral; of 1851 men referred to rescreening, 31 and 50 were referred to elective surgery as a consequence of rescreening once or for life, respectively.

\section{Validation of model}

We used the numbers of predicted key events to validate the model against external trial observations. ${ }^{2}$ Although no statistical differences were seen between model predictions and trial observations, we did note-as expected-that the model was very conservative with fewer ruptures and fewer symptomatic patients, ultimately leading to fewer aneurysm related deaths. Therefore, the number of detected aneurysms due to screening was lower in the model than those observed in the trial, whereas the model predictions for rescreening were within the trial observed levels (tables 1 to 5 in the web appendix). Overall, the differences between model predictions and trial observations reflected a conservative, modern model. In particular, a higher prevalence than the overall $3.3 \%$ used to inform the model would lead to significantly higher event rates.

The model assumed no correlations between the input distributions for the probabilistic sensitivity analysis. This assumption could lead to extreme predictions that would bias results. We therefore selected a strategy to assess in detail whether the minimum and maximum numbers of predicted events from the probabilistic sensitivity analysis were within plausible ranges. This seemed to be the case for all key events (table 6 in the web appendix).

\section{Base case cost effectiveness}

Table $4 \Downarrow$ shows the average costs and QALYs for the four strategies and the probabilities for cost effectiveness, in view of the threshold values of willingness to pay per QALY at $£ 20$ 000 and $£ 30000$, respectively. The net present value of the gain from screening compared with not screening was 11434 QALYs per 100000 men screened. The corresponding gain from screening twice was 84 QALYs, and if rescreening was repeated for lifetime, an additional 20 QALYs would be gained.

Cost of screening was estimated at $£ 6.34$ million per 100000 men screened, whereas the incremental costs of additional screening were limited to about $£ 843000$ for rescreening once and an additional $£ 603000$ for lifetime rescreening. Altogether, these values represented incremental cost effectiveness ratios of $£ 555$ for screening versus no screening, $£ 10013$ for screening twice versus screening once, and $£ 29680$ for lifetime rescreening versus screening twice. It was clearly cost effective to screen as opposed to not screen, but the optimal choice of rescreening strategy remained uncertain, as indicated by the 


\section{Box: Specified scenarios for the sensitivity analysis}

1) Assuming a less aggressive epidemiology, which could represent future healthcare with modern medical prevention, will ultimately reduce the prevalence or growth rates further. Prevalence was reduced by $50 \%$ (to $1.63 \%$ overall), and growth rates were all reduced by $25 \%$.

2) Assuming a temporary effect of being followed for aneurysm growth on the quality of life of individuals. For patients with aneurysms of $30-55 \mathrm{~mm}$ diameter, the age specific quality of life was reduced by $6 \%{ }^{30}$ When patients exited the follow-up states (figure), quality of life was assumed to normalise to the model's base case level.

3) The organisational design of a screening programme includes a trade-off between local provision (possibly leading to high attendance rates) and economies of scale and scope. The present organisational design proposal seem to be more centralised (and cheaper) than the programme currently being implemented in England. We tested the influence of a higher screening cost per person invited to screening of $£ 30$ ( $€ 37 ; \$ 47)$ and a higher attendance rate at the initial screening of $80 \%$. Both levels corresponded to those observed in the English context. ${ }^{6}$

4) The patient perspective is often neglected in screening models, as is an overall societal perspective in which production losses could occur when workers leave their jobs to attend health programmes. We tested a societal perspective, including average transportation costs of $£ 7.96$ per participant, private time costs of $£ 7.85$ per participant, and production loss of $£ 2.69$ per participant. ${ }^{31}$

probabilities for cost effectiveness being almost equally distributed among the three screening strategies (with a small overweight in favour of the rescreening strategies).

\section{Sensitivity analyses}

We tested four alternative scenarios to assess the sensitivity of findings to alternative assumptions. None of the scenarios significantly altered the cost effectiveness of some form of screening compared with no screening, as the probability for cost effectiveness of screening did not drop below 0.91 (table $5 \Downarrow)$. In relation to the optimal rescreening strategy, the base case probabilities for cost effectiveness were relatively close across the alternatives and for that reason, even marginal impacts of alternative assumptions could have affected the results. In particular, the alternative scenario 2, which tested for the influence of a temporary reduction in quality of life associated with enrolment in annual or biannual follow-up regimens, had a considerable effect on the QALY gain of rescreening. For lifetime rescreening, the QALY gain even became negative and led to the domination of the other screening strategies.

\section{Discussion}

\section{Principal findings}

This study confirms the cost effectiveness of screening versus not screening men for abdominal aortic aneurysms, and further assesses the attractiveness of rescreening. At a threshold of $£ 20$ 000 per QALY, rescreening once seems to be the optimal strategy with an average incremental cost effectiveness ratio of $£ 10013$. However, substantial uncertainty surrounds this ratio, and with an average incremental cost effectiveness ratio of lifetime rescreening at $£ 29680$, the optimal rescreening strategy is indeterminate. For those enrolled in follow-up for aneurysms below the surgical threshold, the alternative scenario analysis showed that a negative effect on quality of life might alter the attractiveness of rescreening. Clear evidence for such an effect on quality of life is lacking, which, along with more detailed evidence for growth rates of aneurysms, would greatly reduce the decision uncertainty.

\section{Strengths and limitations}

This work is, to the best of our knowledge, the first to assess the cost effectiveness of rescreening. It also considers the current, modern world in which medical prevention has improved markedly, endovascular aortic repair is now commonly used, symptoms are a common indication for surgery, and the disease process is known to be more complex than a three state model. Finally, a major strength of our methodology was the extensive tracking of key events and subsequent microsimulation analyses. To the best of our knowledge, this is the first example of this technique in the abdominal aortic aneurysm literature.

The model included an effect of screening on non-aneurysm related mortality, which has been discussed for years but not included in previous models. The theoretical expectation that screening should decrease non-aneurysm related mortality is multifaceted. Firstly, the strict terminology of aneurysm related mortality and non-aneurysm related mortality is somewhat notional because, in practice, mortality is rarely attributable to a single cause and therefore could be misclassified. Furthermore, it cannot be ruled out that screening actually leads to prophylactic actions in patients. Regardless of the explanation, failing to allow a potential influence of screening on the total mortality biases the results against screening. The magnitude of such biases was previously demonstrated after four years of follow-up in the Multicentre Aneurysm Screening Study, where a $2 \%$ risk reduction in mortality due to ischemic heart disease was observed in the screening group. ${ }^{12}$

A major challenge for this type of research is that the growth and rupture rates are key parameters for predicting the disease course. According to European clinical guidelines, ${ }^{32}$ aneurysms with aortic diameters of $55 \mathrm{~mm}$ or more are referred for surgical evaluation once they are detected, and consequently, there are no original studies for growth and rupture in patients with aneurysms in the general population. The limited evidence consists of patients with contraindications for surgery. In the present study, we assumed a linear growth function based on observations of smaller aneurysms and did a systematic literature review to determine rupture rates. The linear assumption for the growth rate could have been an overly conservative assumption for larger aneurysms. ${ }^{33}$

An important assumption of the economic evaluation methodology is that all feasible strategies are considered. The present model included four strategies, although other strategies could be relevant also-such as screening at another constant interval than five years or screening at non-constant intervals. The rationale for the present set-up was to contrast strategies that have figured in recent clinical management debates while maintaining a reasonable degree of complexity in the model. The strategies left out of the model can be seen as intermediate or mixed strategies, and generally the cost effectiveness of these strategies will be expected to fall between the "raw" strategies they are composed of.

In terms of generalisability of the present findings to the United Kingdom, a concern would be that Danish item costs were used. The cost effectiveness of screening was obviously driven by costs for the screening and surgeries. To a large extent however, the benefit of screening is that acute surgery is replaced by elective surgery and therefore the cost difference between the two types of surgery rather than the absolute costs is relevant 
to assess. Based on previously reported UK item costs, the screening cost seems to be higher in the UK than in Denmark, whereas the opposite seems to be the case for the cost difference between the two types of surgeries. Therefore, the overall costs of screening versus no screening could be underestimated for the UK setting, whereas the overall costs of rescreening versus no rescreening could be overestimated.

\section{Comparison with other studies}

The cost effectiveness of rescreening has not been studied previously. Several studies have concluded that screening is cost effective compared with no screening over a lifetime..$^{5-10}$ This result is confirmed in the current model, which shares its two baseline strategies with the literature, although the updated epidemiology would lead us to expect a lower QALY gain of screening at baseline than that seen in previous studies. On the other hand, inclusion of the effect of screening on non-aneurysm related mortality would lead us to expect a higher total QALY gain. Our base case analysis estimated the total gain at about 0.11 QALYs between screening and no screening (table 4). In other recent studies, the estimated gains ranged from 0.02 (in a Canadian model, two Swedish models, and an English model) to 0.05 (in a North American model), and 0.05 and 0.07 (in the Dutch and Norwegian components of a combined model, respectively). ${ }^{5-10}$ Differences between present and previously estimated gains of QALYs can be attributed mainly to the effect of screening on non-aneurysm related mortality and to increased life expectancy, reduced prevalence of aneurysms, higher rate for incidental detection, and a more detailed modelling of the disease process.

\section{Future research and policy implications}

The effect of screening on quality of life is critical to the decision scenario. Although temporary effects might be associated with men being offered and participating in screening, more influential effects could stem from detection of aneurysms. Patients with detected aneurysms eligible for referral to elective or acute surgery could have temporary anxiety until successful treatment is completed, whereas it has been shown that patients return to the population norm for quality of life after treatment. ${ }^{34}$ For patients detected with smaller aneurysms, there will be a (lengthy) follow-up period in which their quality of life could be suppressed owing to the uncertainty about their disease course. We conducted a systematic literature review and identified no studies appropriately estimating the effect of such uncertainty. ${ }^{22}$ Since our results were sensitive to reductions in quality of life during follow-up, this research goal is important for future studies.

Further research on growth and rupture rates would have a positive effect on the decision uncertainty. Although most patients are referred for surgery once an abdominal aortic aneurysm is detected, it should be possible to observe the time from detection to planned surgery, optimally on a national scale and in a healthcare system in which the waiting time is significant. In addition, the probabilistic modelling framework allows for analysis of the value of reducing the uncertainty surrounding particular parameters, which can help inform future research priorities, since it was not a formal part of the present analysis. ${ }^{35}$

This study has policy relevance for two different scenarios. In Denmark, where no national guidance has been issued, it suggests that there is a high probability that screening will be cost effective. In England and Scotland, where screening is currently being implemented, this study supports further consideration of rescreening, at least once.

We thank the key stakeholders and field experts who were invited to observe the development of the model (Anders Green, Ole Andersen, Henrik Sillesen, and Jan Sørensen), who provided meaningful contributions at different stages of the project; the Danish society for vascular surgery, which provided ad hoc estimates from the Danish vascular registry.

Contributors: JSL and RS conceived the study. The model was specified in close collaboration between RS (health economist who specified the methodology, built the model, and defined the parameters) and JSL and $\mathrm{JL}$ (clinical experts who guided the definition of strategies and conducted literature searches or original analysis of primary data). RS conducted the analysis, drafted the manuscript, and is responsible for the overall content as guarantor. JSL and JL participated in a critical revision of the manuscript, and all authors approved the final version. Funding: The Health Research Fund of Central Denmark Region and the Research Fund of Viborg Hospital funded the work. The sponsors had no role in the study design; in the collection, analysis, and interpretation of data; in the writing of the report; and in the decision to submit the paper for publication.

All authors have completed the Unified Competing Interest form at www. icmje.org/coi_disclosure.pdf (available on request from the corresponding author) and declare: support from the Health Research Fund of Central Denmark Region and the Research Fund of Viborg Hospital; JSL was sponsored by the 7th European Framework Programme

(Health-2007-2.4.2-2; project title "Fighting aneurysmal disease"; grant agreement no 200647); no other relationships or activities that could appear to have influenced the submitted work.

Ethical approval: No ethical approval was required. The study was approved by the Danish Data Protection Agency.

Data sharing: No additional data are available (the model builds on secondary data).

Wilson J, Jungner G. Principles and practice of screening for disease. WHO, 1968

2 Lindholt JS, Sørensen J, Søgaard R, Henneberg EW. Long-term benefit and cost-effectiveness analysis of screening for abdominal aortic aneurysms from a randomized controlled trial. Br J Surg 2010;97:826-34.

3 Thompson SG, Ashton HA, Gao L, Scott RA. Screening men for abdominal aortic aneurysm: 10 year mortality and cost effectiveness results from the randomised Multicentre Aneurysm Screening Study. BMJ 2009;338:b2307.

4 Brownsword R, Earnshaw JJ. The ethics of screening for abdominal aortic aneurysm in men. J Med Ethics 2010;36:827-30.

5 Henriksson M, Lundgren F. Decision-analytical model with lifetime estimation of costs and health outcomes for one-time screening for abdominal aortic aneurysm in 65-year-old men. Br J Surg 2005;92:976-83.

6 Kim LG, Thompson SG, Briggs AH, Buxton MJ, Campbell HE. How cost-effective is screening for abdominal aortic aneurysms? J Med Screen 2007;14:46-52.

7 Montreuil B, Brophy J. Screening for abdominal aortic aneurysms in men: a Canadian perspective using Monte Carlo-based estimates. Can J Surg 2008:51:23-34.

8 Silverstein MD, Pitts SR, Chaikof EL, Ballard DJ. Abdominal aortic aneurysm (AAA): cost-effectiveness of screening, surveillance of intermediate-sized AAA, and management of symptomatic AAA. Proc (Bayl Univ Med Cent) 2005;18:345-67.

9 Spronk S, van Kempen BJ, Boll AP, Jorgensen JJ, Hunink MG, Kristiansen IS. Cost-effectiveness of screening for abdominal aortic aneurysm in the Netherlands and Norway. Br J Surg 2011;98:1546-55.

10 Wanhainen A, Lundkvist J, Bergqvist D, Bjorck M. Cost-effectiveness of different screening strategies for abdominal aortic aneurysm. J Vasc Surg 2005;41:741-51.

11 Søgaard R, Lindholt J. Evidence for the credibility of health economic models for health policy decision-making: a systematic literature review of screening for abdominal aortic aneurysms. J Health Serv Res Policy 2012;17:44-52.

12 Ashton HA, Buxton MJ, Day NE, Kim LG, Marteau TM, Scott RA, et al. The Multicentre Aneurysm Screening Study (MASS) into the effect of abdominal aortic aneurysm screening on mortality in men: a randomised controlled trial. Lancet 2002;360:1531-9.

13 Walley $T$, Folino-Gallo $P$, Stephens $P$, Van Ganse $E$. Trends in prescribing and utilization of statins and other lipid lowering drugs across Europe 1997-2003. Br J Clin Pharmacol 2005;60:543-51.

14 Metcalfe D, Holt PJ, Thompson MM. The management of abdominal aortic aneurysms. BMJ 2011;342:d1384.

15 Levin DC, Rao VM, Frangos AJ, Parker L, Sunshine JH. Recent trends in utilization of vascular ultrasound among radiologists, surgeons, cardiologists, and other physicians. $J$ Am Coll Radiol 2007;4:125-7.

16 Kent KC. Endovascular aneurysm repair-is it durable? N Engl J Med 2010;362:1930-1.

17 Powell JT, Sweeting MJ, Brown LC, Gotensparre SM, Fowkes FG, Thompson SG. Systematic review and meta-analysis of growth rates of small abdominal aortic aneurysms. Br J Surg 2011;98:609-18. 


\section{What is already known on this topic}

One-off screening of men aged 65 years for abdominal aortic aneurysm is known to be cost effective at conventional thresholds for cost effectiveness

National screening programmes are currently being implemented in England and Scotland, but many other European countries have refrained from issuing national guidance on screening

In addition to the current screening programmes, rescreening those patients with an aortic diameter near the defined aneurysm size $(25-29 \mathrm{~mm})$ is currently being debated

\section{What this study adds}

This study presents a comprehensive decision model predicting that, if screening is repeated once or repeated every five years for their lifetime, an aneurysm will be detected in $\mathbf{4 5 2}$ or 794 men per 100000 initially screened, respectively

Rescreening seems to be associated with average incremental cost effectiveness ratios of $£ 10013$ for rescreening once and $£ 29680$ for lifetime rescreening, but considerable decision uncertainty exists between the two strategies

18 Vardulaki KA, Prevost TC, Walker NM, Day NE, Wilmink AB, Quick CR, et al. Growth rates and risk of rupture of abdominal aortic aneurysms. Br J Surg 1998;85:1674-80.

19 NHS. Abdominal aortic aneurysm screening programme. NHS, 2011.

$20 \mathrm{CMO}$ and Public Health Directorate. Scottish abdominal aortic aneurysm screening programme. The Scottish Government, 2010

21 Grøndal N, Søgaard R, Henneberg EW, Lindholt JS. The Viborg vascular (VIVA) screening trial of 65-74 year old men in the central region of Denmark: study protocol. Trials 2010;11:67

22 Søgaard R, Laustsen J, Lindholt J. Modelling cost-effectiveness of screening for abdominal aortic aneurysms. Technical report. University of Southern Denmark, 2011.

23 Brown PM, Zelt DT, Sobolev B. The risk of rupture in untreated aneurysms: the impact of size, gender, and expansion rate. J Vasc Surg 2003;37:280-4.

24 Lederle FA, Johnson GR, Wilson SE, Ballard DJ, Jordan WD Jr, Blebea J, et al. Rupture rate of large abdominal aortic aneurysms in patients refusing or unfit for elective repair. JAMA 2002;287:2968-72.

25 Sørensen J, Davidsen M, Gudex C, Pedersen KM, Brønnum-Hansen H. Danish EQ-5D population norms. Scand J Public Health 2009;37:467-74.

26 Lindholt JS, Sørensen J. [Hospital costs for abdominal aortic aneurysm surgery.] Ugeskr Laeger 2010;172:2206-12.

27 Lindholt JS, Norman PE. Meta-analysis of postoperative mortality after elective repair of abdominal aortic aneurysms detected by screening. Br J Surg 2011;98:619-22.

28 Lindholt JS, Juul S, Fasting H, Henneberg EW. Screening for abdominal aortic aneurysms: single centre randomised controlled trial. BMJ 2005:330:750.

29 Scott RA, Thompson SG. Screening, surgical repair, and the management of abdominal aortic aneurisms. J Med Screen 2005;12:57-8.
30 Lindholt JS, Vammen S, Fasting H, Henneberg EW. Psychological consequences of screening for abdominal aortic aneurysm and conservative treatment of small abdominal aortic aneurysms. Eur J Vasc Endovasc Surg 2000;20:79-83.

31 Vammen S, Lindholt JS, Juul S, Henneberg EW, Fasting H. Screening for abdominal aortic aneurysms: an analysis of the private and indirect costs in a hospital-based screening program. Int J Angiol 2001;10:246-9.

32 Moll FL, Powell JT, Fraedrich G, Verzini F, Haulon S, Waltham M, et al. Management of abdominal aortic aneurysms clinical practice guidelines of the European society for vascular surgery. Eur J Vasc Endovasc Surg 2011;41(suppl 1):S1-58.

33 Sweeting MJ, Thompson SG. Making predictions from complex longitudinal data, with application to planning monitoring intervals in a national screening programme. In: Unit MB, ed. Technical report. MRC Biostatistics Unit, 2010.

34 Tambyraja AL, Fraser SC, Murie JA, Chalmers RT. Quality of life after repair of ruptured abdominal aortic aneurysm. Eur J Vasc Endovasc Surg 2004;28:229-33.

35 Ades AE, Lu G, Claxton K. Expected value of sample information calculations in medical decision modeling. Med Decis Making 2004:24:207-27.

Accepted: 21 May 2012

\section{Cite this as: BMJ 2012;345:e4276}

This is an open-access article distributed under the terms of the Creative Commons Attribution Non-commercial License, which permits use, distribution, and reproduction in any medium, provided the original work is properly cited, the use is non commercial and is otherwise in compliance with the license. See: http://creativecommons.org/licenses/by$\mathrm{nc} / 2.0 /$ and http://creativecommons.org/licenses/by-nc/2.0/legalcode. 


\section{Tables}

\section{Table 1| Parameter estimates}

\begin{tabular}{|c|c|c|c|}
\hline Description & Mean & Distribution* & Source \\
\hline Prevalence or disease distribution (\%) & 3.3 & $\begin{array}{c}\text { Dirichlet }(94.11 ; 2.64 ; 2.71 ; 0.22 ; 0.12 ; 0.08 ; \\
0.06 ; 0.06)\end{array}$ & VIVA/Viborg trials ${ }^{2,21}$ \\
\hline \multicolumn{4}{|l|}{ Attendance (\%) } \\
\hline At initial screening & 75 & Beta $(24615,18378)$ & \multirow[t]{3}{*}{-} \\
\hline At rescan & 87 & Beta $(286,248)$ & \\
\hline At follow-up & 89 & Beta $(169,150)$ & \\
\hline Patients undergoing endovascular repair (\%) & 41 & Beta $(5146,2122)$ & Danish vascular registry \\
\hline \multicolumn{4}{|l|}{ Mortality risk of surgery (\%) } \\
\hline Elective repair & 3.0 & Beta $(1689,50)$ & - \\
\hline Acute repair & 8.6 & Beta $(304,26)$ & - \\
\hline Acute repair after rupture & 39 & Beta $(832,322)$ & - \\
\hline \multicolumn{4}{|l|}{ Patients with symptoms (\%) } \\
\hline $50-54 \mathrm{~mm}$ & 0.1 & Beta $(1472,2)$ & \multirow[t]{5}{*}{-} \\
\hline $55-59 \mathrm{~mm}$ & 1.4 & Beta $(1472,20)$ & \\
\hline $60-69 \mathrm{~mm}$ & 1.5 & Beta $(1472,22)$ & \\
\hline $70-79 \mathrm{~mm}$ & 1.7 & Beta $(1472,25)$ & \\
\hline$\geq 80 \mathrm{~mm}$ & 4.8 & Beta $(1472,70)$ & \\
\hline Contraindication surgical repair (\%) & 8.2 & Beta $(487,40)$ & $\begin{array}{l}\text { Vardulaki et al } 2002,{ }^{18} \text { Lindholt et al } \\
2005,{ }^{28} \text { Scott et al } 2005^{29}\end{array}$ \\
\hline \multicolumn{4}{|l|}{ Growth to next state (annual rate) } \\
\hline $25-29 \mathrm{~mm}$ & 0.07 & Beta $(648,48)$ & VIVA/Viborg trials ${ }^{2,21}$ \\
\hline $30-49 \mathrm{~mm}$ & 0.09 & Beta $(363,33)$ & - \\
\hline $50-54 \mathrm{~mm}$ & 0.62 & Fixed & $\begin{array}{l}\text { Assumption based on extrapolation of } \\
\text { data from VIVA/Viborg trials }\end{array}$ \\
\hline $55-59 \mathrm{~mm}$ & 0.67 & Fixed & 一 \\
\hline $60-69 \mathrm{~mm}$ & 0.39 & Fixed & - \\
\hline $70-79 \mathrm{~mm}$ & 0.44 & Fixed & - \\
\hline \multicolumn{4}{|l|}{ Rupture (annual rate) } \\
\hline $50-54 \mathrm{~mm}$ & 0.01 & Beta $(607,6)$ & \multirow{5}{*}{$\begin{array}{l}\text { Lederle et al } 2002,{ }^{24} \text { Brown et al } \\
2003^{23}\end{array}$} \\
\hline $55-59 \mathrm{~mm}$ & 0.10 & Beta $(62,6)$ & \\
\hline $60-69 \mathrm{~mm}$ & 0.11 & Beta $(130,14)$ & \\
\hline $70-79 \mathrm{~mm}$ & 0.12 & Beta $(73,9)$ & \\
\hline$\geq 80 \mathrm{~mm}$ & 0.39 & Beta $(57,22)$ & \\
\hline Incidental detection of $\geq 55 \mathrm{~mm}(\%)$ & 12 & Beta $(1977,232)$ & $\begin{array}{l}\text { Danish vascular registry, VIVA/Viborg } \\
\text { trials, }{ }^{2,21} \text { Danish national patient registry }\end{array}$ \\
\hline Reaching hospital alive with rupture (\%) & 45 & Beta $(6954,3148)$ & $\begin{array}{l}\text { Danish vascular registry, Danish } \\
\text { national death-cause registry }\end{array}$ \\
\hline \multicolumn{4}{|l|}{ Costs ( $£$ in 2010) } \\
\hline Endovascular repair & 17377 & Gamma $(4,0)$ & \multirow{6}{*}{$\begin{array}{l}\text { Lindholt et al } 2010,{ }^{2} \text { Lindholt and } \\
\text { Sørensen } 2010^{26}\end{array}$} \\
\hline Follow-up scan & 205 & Gamma $(248,1)$ & \\
\hline Open acute repair & 19778 & Gamma $(10,0)$ & \\
\hline Open acute repair after rupture & 25733 & Gamma $(4,0)$ & \\
\hline Open elective repair & 11108 & Gamma $(146,0)$ & \\
\hline Screening per invitee & 20 & Gamma $(400,20)$ & \\
\hline \multicolumn{4}{|l|}{ Excess mortality (odds ratio) } \\
\hline $30-49 \mathrm{~mm}$ & 1.46 & Fixed & \multirow{2}{*}{$\begin{array}{l}\text { Danish vascular registry, VIVA/Viborg } \\
\text { trials, }{ }^{2,21} \text { Danish national mortality } \\
\text { - statistics }\end{array}$} \\
\hline $50-54 \mathrm{~mm}$ & 1.70 & Fixed & \\
\hline
\end{tabular}




\section{Table 1 (continued)}

\begin{tabular}{|c|c|c|c|}
\hline Description & Mean & Distribution* & Source \\
\hline$>55 \mathrm{~mm}$ & 2.15 & Fixed & \\
\hline Inoperable aneurysm & 2.57 & Fixed & \\
\hline $\begin{array}{l}\text { Reduced non-AAA related mortality in screened } \\
\text { men (odds ratio) }\end{array}$ & 0.98 & Normal $(0.98,0.02)$ & Søgaard et al $2011^{22}$ \\
\hline $\begin{array}{l}\text { Reduced postelective mortality in screened men } \\
\text { (odds ratio) }\end{array}$ & 0.37 & Normal $(0.37,0.14)$ & Lindholt and Norman $2011^{27}$ \\
\hline
\end{tabular}

$\mathrm{AAA}=$ abdominal aortic aneurysm; $\mathrm{mm}=$ aortic diameter in millimetres.

*Values refer to prevalence (Dirichlet distribution); the number surveyed and the number of events (beta distribution); the shape and the scale of the distribution (gamma distribution); and the mean and standard deviation (normal distribution). 
Table 2| Predicted numbers of key events under four alternative strategies for the management of abdominal aortic aneurysms (AAA). Data are number or percentage unless stated otherwise

\begin{tabular}{|c|c|c|c|c|c|c|c|}
\hline & \multicolumn{4}{|c|}{ No of events per 100000 men } & \multicolumn{3}{|c|}{ Relative risk reduction (\%) } \\
\hline & No screening & Screening once & Screening twice & $\begin{array}{l}\text { Lifetime } \\
\text { screening }\end{array}$ & $\begin{array}{l}\text { Screening once } v \\
\text { no screening }\end{array}$ & $\begin{array}{l}\text { Screening twice } v \\
\text { screening once }\end{array}$ & $\begin{array}{c}\text { Lifetime screening } \\
\text { v screening twice }\end{array}$ \\
\hline \multicolumn{8}{|l|}{ Detection } \\
\hline Incidental & 934 & 646 & 618 & 569 & -31 & -4 & -8 \\
\hline By screening & 0 & 2469 & 2469 & 2469 & Not applicable & Not applicable & Not applicable \\
\hline By rescreening & 0 & 0 & 452 & 794 & Not applicable & Not applicable & Not applicable \\
\hline Total & 934 & 3115 & 3539 & 3832 & 234 & 14 & 8 \\
\hline \multicolumn{8}{|l|}{ Elective surgery } \\
\hline Endovascular & 364 & 604 & 632 & 658 & 66 & 5 & 4 \\
\hline Open & 497 & 786 & 864 & 872 & 58 & 10 & 1 \\
\hline Total & 861 & 1390 & 1496 & 1530 & 61 & 8 & 2 \\
\hline \multicolumn{8}{|l|}{ Acute surgery } \\
\hline For rupture & 492 & 305 & 297 & 294 & -38 & -3 & -1 \\
\hline For symptoms & 118 & 77 & 66 & 66 & -35 & -14 & 0 \\
\hline Total & 610 & 382 & 363 & 360 & -37 & -5 & -1 \\
\hline Rupture & 1069 & 719 & 692 & 682 & -33 & -4 & -1 \\
\hline \multicolumn{8}{|l|}{ Mortality } \\
\hline AAA related & 788 & 538 & 520 & 511 & -32 & -3 & -2 \\
\hline Non-AAA related & 99212 & 99459 & 99477 & 99486 & 0 & 0 & 0 \\
\hline Total & 100000 & 99997 & 99997 & 99997 & 0 & 0 & 0 \\
\hline \multicolumn{8}{|l|}{ Age at death (years) } \\
\hline AAA related & 73.50 & 74.33 & 74.37 & 74.30 & Not applicable & Not applicable & Not applicable \\
\hline Non-AAA related & 80.92 & 81.09 & 81.09 & 81.09 & Not applicable & Not applicable & Not applicable \\
\hline Total & 80.86 & 81.05 & 81.05 & 81.06 & Not applicable & Not applicable & Not applicable \\
\hline
\end{tabular}

Predictions based on microsimulation of 100000 men in a Markov model. 
Table 3| Predicted numbers of key events in the rescreening strategies for men with an aortic diameter of 25-29 mm at initial screening

\begin{tabular}{lcccc} 
& \multicolumn{2}{c}{ No of events per $\mathbf{1 0 0} \mathbf{0 0 0}$ men } & \\
\cline { 2 - 4 } & Screening twice & Lifetime screening & Relative risk reduction (\%) \\
Referred for rescan programme & 1851 & 2978 & 61 \\
\hline Incidental detection during programme & 8 & 16 & 100 \\
\hline Symptoms during programme & 0 & 0 & Not applicable \\
\hline Rupture during programme & 6 & 9 & 50 \\
\hline Dead during programme & 193 & 622 & 222 \\
\hline Available for rescan & 1644 & Not applicable & Not applicable \\
\hline Attended rescan & 1418 & 2565 & 81 \\
\hline Findings at rescan & & & -35 \\
\hline No growth & 966 & 624 & 79 \\
\hline Diameter $30-49 \mathrm{~mm}$ & 364 & 653 & 60 \\
\hline Diameter $50-54 \mathrm{~mm}$ & 57 & 91 & 61 \\
\hline Diameter $\geq 55 \mathrm{~mm}$ & 31 & 50 & 76 \\
\hline Aneurysms detected & 452 & 794 & \\
\hline
\end{tabular}

Predictions based on microsimulation of 100000 men in a Markov model. 
Table 4| Base case analysis

\begin{tabular}{lccccc} 
& & & & & \multicolumn{2}{c}{ Probability of cost effectiveness $^{*}$} \\
& QALYs (mean) & Costs (£ mean) & $\begin{array}{c}\text { Incremental cost } \\
\text { effectiveness ratio }(£)\end{array}$ & £20 000 & £30 000 \\
\cline { 5 - 7 } No screening & 11.8474 & $£ 230$ & Not applicable & 0.08 & 0.08 \\
\hline Screening once & 11.9618 & $£ 293$ & 555 & 0.27 & 0.26 \\
\hline Screening twice & 11.9626 & $£ 301$ & 10013 & 0.33 & 0.32 \\
\hline Lifetime screening & 11.9628 & $£ 308$ & 29680 & 0.33 & 0.34 \\
\hline
\end{tabular}

*Probability that the strategy is more cost effective than the other three strategies, in view of the particular maximum willingness to pay £20 000 or £30 000 per additional quality adjusted life year. 


\begin{tabular}{|c|c|c|c|c|c|}
\hline \multicolumn{6}{|c|}{ Table 5| Results of alternative scenario analysis } \\
\hline & \multirow[b]{2}{*}{ QALYs (mean) } & \multirow[b]{2}{*}{ Costs ( $£$ mean) } & \multirow{2}{*}{$\begin{array}{l}\text { Incremental cost } \\
\text { effectiveness ratio }(£)\end{array}$} & \multicolumn{2}{|c|}{ Probability of cost effectiveness ${ }^{*}$} \\
\hline & & & & $£ 20000$ & $£ 30000$ \\
\hline \multicolumn{6}{|c|}{ Scenario 1 (reduced prevalence and growth rates) } \\
\hline No screening & 11.9280 & 96 & Not applicable & 0.09 & 0.09 \\
\hline Screening once & 12.0375 & 134 & 354 & 0.32 & 0.31 \\
\hline Screening twice & 12.0378 & 138 & 11435 & 0.31 & 0.31 \\
\hline Lifetime screening & 12.0379 & 140 & 38814 & 0.29 & 0.29 \\
\hline \multicolumn{6}{|c|}{ Scenario 2 (negative effect on quality of life while enrolled in follow-up) } \\
\hline No screening & 11.8474 & 230 & Not applicable & 0.09 & 0.08 \\
\hline Screening once & 11.9568 & 293 & 580 & 0.40 & 0.39 \\
\hline Screening twice & 11.9569 & 301 & 88292 & 0.32 & 0.32 \\
\hline Lifetime screening & 11.9567 & 308 & Dominated strategy $\dagger$ & 0.20 & 0.21 \\
\hline \multicolumn{6}{|c|}{ Scenario 3 (local provision (higher attendance rate and programme costs)) } \\
\hline No screening & 11.8502 & 228 & Not applicable & 0.09 & 0.09 \\
\hline Screening once & 11.9638 & 292 & 565 & 0.25 & 0.24 \\
\hline Screening twice & 11.9647 & 301 & 8548 & 0.31 & 0.30 \\
\hline Lifetime screening & 11.9650 & 307 & 22744 & 0.35 & 0.37 \\
\hline \multicolumn{6}{|c|}{ Scenario 4 (societal costing perspective) } \\
\hline No screening & 11.8473 & 230 & Not applicable & 0.07 & 0.07 \\
\hline Screening once & 11.9621 & 307 & 668 & 0.26 & 0.26 \\
\hline Screening twice & 11.9629 & 315 & 10560 & 0.30 & 0.29 \\
\hline Lifetime screening & 11.9631 & 322 & 24370 & 0.37 & 0.38 \\
\hline
\end{tabular}




\section{Figure}

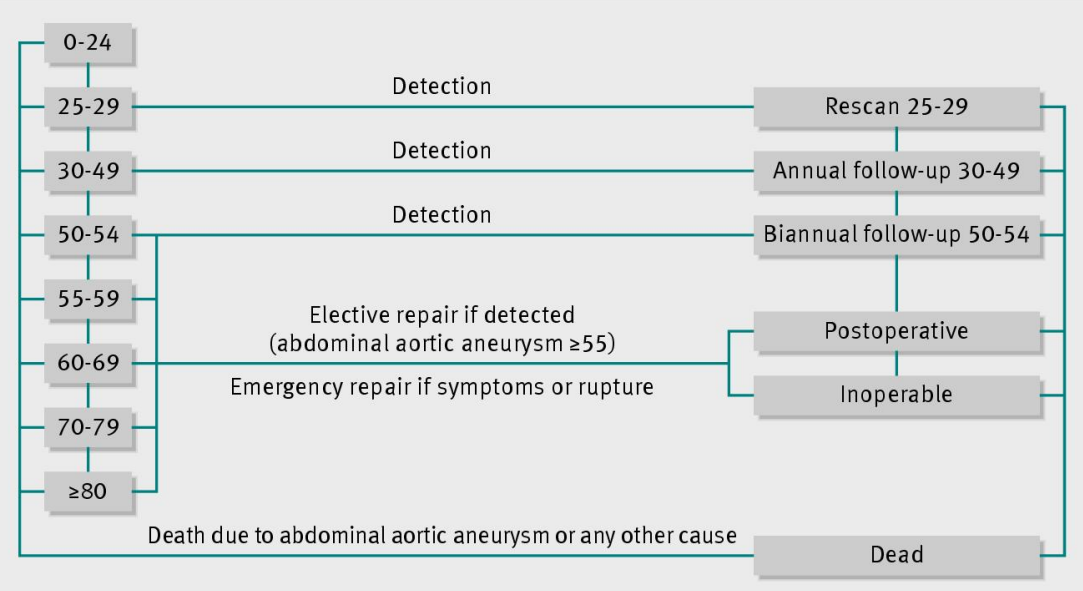

Fig 1 Markov model for the course of abdominal aortic aneurysms. Diagram is a simplified presentation of the disease history and relevant modalities for intervention. Eight boxes to the left of the diagram=starting states; numbers=abdominal aortic diameter $(\mathrm{mm})$. Men either remain in their current state or move to a neighbouring state, following the connecting lines. Detection of aneurysms can be incidental or follow on from systematic screening 